\title{
Communication and Coordination
}

\author{
John H. Miller* Scott Moser ${ }^{\dagger}$
}

February 25, 2003

\begin{abstract}
Remarkable levels of coordination are observed among social agents; yet the exact mechanisms by which such agents coordinate are not well understood. Here we examine the role of communication in achieving coordination - in particular, does endowing agents with the ability to communicate lead to more favorable outcomes? To pursue this question we employ an adaptive model of strategically communicating agents (Miller et al. [7]) playing the Stag Hunt game.

We find that communication plays a key role in the ability of agents to reach and maintain superior coordination. In the absence of communication, agents tend to get trapped at the inferior coordination point. However, once agents reach a particular strategic threshold, sending even a priori meaningless messages serves to increase the likelihood that the population will coordinate on the superior outcome. While the system spends the majority of its time with well-coordinated behavior, it is not static - such periods are often punctuated by brief transitions in which the system switches to the alternative coordination point. We analyze the various mechanisms that account for this dynamic behavior and find that there are a few critical pathways by which the system transitions from one coordination point to another. Communication plays a critical, yet short-lived, role in one key pathway.

Our analysis suggests that giving agents the ability to communicate even a priori meaningless messages may promote the emergence of a rich, and often robust, "ecology" of behaviors that allows agents to achieve new, and in this case superior, outcomes.
\end{abstract}

\footnotetext{
*Carnegie Mellon University and the Santa Fe Institute, email: miller@santafe.edu. Miller's work was supported, in part, by core funding from the Santa Fe Institute.

†Carnegie Mellon University, email: smoser@andrew.cmu.edu. Moser's work was supported by NSF IGERT9972762 to Carnegie Mellon University.
} 


\section{Introduction}

Remarkable levels of coordination are observed among social agents; yet the exact mechanisms by which agents coordinate are not fully understood. Here, we examine the role of communication in promoting coordination. To pursue this question we employ an adaptive model of strategically communicating agents (Miller et al. [7]) playing the Stag Hunt game. Our analysis suggests that giving agents the ability to strategically communicate even a priori meaningless messages promotes the emergence of a rich, and often robust, "ecology" of behaviors that allows agents to achieve new, and in this case superior, outcomes.

The system we explore focuses on simple, adaptive agents that can strategically send, and react to, messages to one another. The communication is endogenous, in the sense that the messages are given no initial meaning, and it is only through the decentralized actions and reactions of the individual agents that communication takes on meaning. The model provides a very primitive setting in which we can begin to explore the emergence and impact of communication on adaptive social systems. Clearly more elaborate and realistic models are conceivable, yet the work here explores a useful lower bound on the potential of communication.

We use the Stag Hunt game to provide an interesting context for investigating communication. This simple game models a situation in which two agents would like to coordinate their actions. However, even though one coordination point is superior to the other, both theory and experiment suggest that the inferior coordination point will be chosen in the absence of communication. In the presence of communication, standard theoretical arguments still suggest that superior coordination will not be forthcoming, though the experimental evidence does not always support this conclusion.

Our results suggest that communication, once agents reach a particular strategic threshold, promotes coordination on the superior outcome. Moreover, we find that the likelihood of achieving the better outcome can be tied to both the ability of the agents to implement complicated strategies and the potential of the communicative system. While the system tends to stabilize at the superior coordination point, such coordination is often punctuated by rapid transitions that lead the system to alternative behaviors. We analyze the various mechanisms that account for this dynamic behavior and find that there are a few critical pathways by which the system transitions from one coordination point to another. Communication plays a critical, yet short-lived, role in one key pathway.

The ability of agents to coordinate, whether they be molecules, organisms, or economic entities, is a key feature of a variety of social worlds. Agents actively engaging in communication is one possible mechanism by which coordination can be initiated and maintained. The model developed here suggests that even with simple adaptive mechanisms, endogenous systems of communication can easily arise and facilitate productive coordination. 


\begin{tabular}{|c|c|c|}
\hline & Stag & Hare \\
\hline Stag & 20,20 & $10, C$ \\
Hare & $C, 10$ & 12,12 \\
\hline
\end{tabular}

$20>C \geq 18$

Table 1: Payoffs for the Stag Hunt game.

\section{The Game}

The Stag Hunt game is a useful framework for considering issues of coordination and communication. The payoffs for the version of this game used in the analysis below are given in Table 1. The game is fundamentally one of coordination, whereby the players would like to coordinate their actions. These two coordination points are given by the two pure-strategy Nash equilibria: (Stag,Stag) and (Hare,Hare). Of these two equilibria, the (Stag,Stag) equilibrium is Pareto dominant. Although the (Stag,Stag) equilibrium is socially superior, there is an argument that suggests that the (Hare,Hare) equilibrium is the more probable outcome: if a player is uncertain about how her opponent will play, then playing Hare may give a higher expected payoff than playing Stag. ${ }^{1}$ Harsani and Selton [6] formalized this argument using the notion of risk-dominant equilibria, which, in the case of the game above, suggests that (Hare,Hare) should be the outcome.

Pre-play communication has the potential of improving coordination in the Stag Hunt game. If the issue is uncertainty about the move of one's opponent, then pre-play communication could be used to resolve this uncertainty and achieve the better equilibrium. However, note that regardless of what move a player is about to choose, she always prefers that her opponent play Stag. Aumann [1] conjectured that because of this feature, pre-play communication would be uninformative and hence ineffective in promoting the play of (Stag,Stag).

Although the logic is straightforward, the claim that pre-play communication will not affect the outcome of the Stag Hunt game seems behaviorally questionable (see, for example, Farell and Rabin [5]). Indeed, experiments with similar games using human subjects suggest that some forms of communication may improve the outcome. For example, Charness [2] found that when one player is allowed to send a single, exogenous signal to the other player, coordination on the superior outcome increased from an average of $16 \%$ (with no signals) to 38$86 \%$ (with signals ${ }^{2}$ ). Cooper et al. [4] and Clark et al. [3] allowed both subjects to each send a single, exogenous signal prior to choosing their moves. Under such a regime, relatively high rates of coordination on the superior outcome were observed, though the amount was very sensitive to the payoff parameters.

\footnotetext{
${ }^{1}$ This effect becomes even stronger as $C$ increases.

${ }^{2}$ The higher value occurred when the agent sending the signal was known to have chosen her move after, versus before, choosing what signal to send.
} 
Thus, the empirical evidence suggests, notwithstanding standard theoretical arguments, that exogenous communication can improve coordination in the Stag Hunt game.

\section{The Model}

Here we explore a simple model of bilateral, endogenous communication following the framework developed by Miller et. al [7]. Communication is endogenous in the sense that the meaning of each symbol must be induced by the agents themselves rather than being tied to some global, a priori definition. Agents, whose behavior is controlled by a simple computer program (a finite automaton), play a bilateral game with every other agent in a finite population and accumulate payoffs. We then invoke a simple evolutionary algorithm in which better performing agents are reproduced in the population with some variation in their strategies. After we create the new population, we iterate the system and begin a new generation.

\subsection{Communication}

In the model, agents engage in rounds of pre-play communication prior to choosing a final move (either Stag or Hare) in the game. During each round, agents are allowed to simultaneously send a priori meaningless tokens to one another from $\{1, \ldots, T\}$. By altering $T$ we can explore how variations in the agent's potential to communicate impact coordination. While agents are able to recognize each distinct token, that is, all agents can differentiate between, say, token 1 and token 2, they impute no other initial meaning to the tokens. Whenever an agent decides on a final move, it begins to emit a $\emptyset$ token, ${ }^{3}$ but provides no other information to its opponent. Once both agents have selected final moves (signaled by the simultaneous emission of $\emptyset$ tokens), a single-shot game is conducted using the payoffs given in Table 1. It is possible for one, or both, of the agents to not choose a final move. In this case "chatting" agents receive a payoff of 0 , while non-chatting partners receive a payoff of 5 .

\section{$3.2 \quad$ Agents}

An agent's behavior is governed by a simple computer program represented by a finite state machine (here, a Moore machine). A finite state machine is composed of a set of states, actions, and transitions. In each state, the agent takes a predetermined action either by sending a communication token $t \in\{1, \ldots, T\}$ or deciding on a final move, $m \in\{$ Stag,Hare $\}$, in which case the agent sends $\emptyset$. In addition to an action, each state also contains a transition mapping from all possible observations of the opponent's actions (here on $\{\emptyset, 1, \ldots, T\}$ ) to a state of the automaton. The automaton enters the state specified by the

\footnotetext{
${ }^{3}$ If an agent decides on a final move prior to any communication, then it simply sends a $\emptyset$ token during each round.
} 


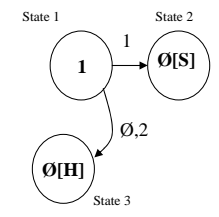

Agent A

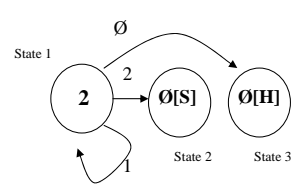

Agent B

$\begin{array}{lllll} & \text { Period 1 } & \text { Period 2 } & \text { Period 3 } & \text { Move } \\ \text { A: } & 1 & \emptyset[\mathrm{H}] & \emptyset[\mathrm{H}] & \mathrm{H} \\ \text { B: } & 2 & 2 & \varnothing[\mathrm{H}] & \mathrm{H}\end{array}$

Figure 1: A sample game.

mapping and proceeds to take the action associated with that state during the next time step. Agents begin each game in State 1 and all actions are exchanged simultaneously. In the experiments below, we fix the maximum number of states $(S)$ that any automaton can use. Note that as the number of states increases, automata are able to embody more complicated strategies (for example, do more counting, branch in more subtle ways, etc.), and thus $S$ is a proxy for the strategic potential of an agent.

Figure 1 illustrates some of the above ideas. Agent $A$ is a three-state automaton that begins in State 1 (represented by the large circle in the diagram) and issues communication token 1 (shown by the label within the circle). If it hears a 1 from its opponent, it follows the transition indicated by the upper arc and proceeds to State 2 (in which case it will play Stag in the game and begin to issue token $\emptyset$ ). Otherwise, the automaton proceeds to State 3 (following the lower arc) where it will choose to play Hare. These latter two states are absorbing in the sense that once the automaton enters them, it will not leave. When Agent $A$ is matched with Agent $B$ in a game, play proceeds as shown in the lower part of the figure. Thus, Agent $B$ initially issues a 2, and on hearing the 1 from Agent $A$ remains in its first state and issues another 2. In the third period, Agent $B$ hears the $\emptyset$ signal from Agent $A$, and this causes it to move to State 3 and choose Hare. Thus, in the fourth period they both have chosen a final move (in this case, both playing Hare). 


\subsection{Evolution of Agents}

The strategies of the agents evolve through a simple adaptive mechanism. Agents are placed in a finite population and play the above game once with every other agent in the population (all the while, accumulating payoff from each game). We then create a new population of agents by reproducing the more successful players. At this stage we also modify some of the reproduced agents so as to introduce new strategies into the population. We then iterate the above procedures and begin a new generation. The resulting system provides a simple laboratory from which to explore the adaptive dynamics of coordination and communication.

More specifically, we initially create a population of thirty random agents. After the agents accumulate payoff, we form a new population of thirty agents through tournament selection. Tournament selection picks two agents at random (with replacement) and places the agent with the higher cumulative payoff into the new population. This selection process is repeated thirty times. Note that this process is biased in favor of the better performing agents, though it does not guarantee that the best agents will be maintained or that the worst ones will be eliminated.

After selection, each agent is modified by mutation with a $50 \%$ probability. If an agent is chosen to be modified, a random state of the machine is picked and with equal probability either that state's action or its transition table is modified. If the state's action is modified, either the existing communication token or the final move decision is replaced (with equal probability over the relevant alternatives). Likewise, if a transition map is modified, a randomly chosen transition is replaced by a randomly selected state.

At the conclusion of selection and modification, a new generation begins. The agents in the new population are again matched for game play, selected for reproduction, and modified as above.

\section{Results}

Despite the stochastic nature of both the selection and mutation processes, the system exhibits surprising regularities. Recall that the primary focus of our analysis is the influence of communication on the ability of the agents to coordinate their actions on (Stag, Stag) play.

There are three parameters of interest to the analysis. The first, $S$, is the maximum number of states allowed in each agent's automaton. As previously discussed, increases in $S$ are associated with the potential for agents to employ more complicated strategies. The second parameter, $T$, is the number of distinct

tokens agents may use to communicate. As $T$ increases agents have the ability to send messages from a larger "language" and hence their communicative potential increases. Finally, we consider the $C$ parameter from the payoff matrix in Figure 1. As $C$ increases, Hare becomes an increasingly attractive action in the single-shot game. Hence, as $C$ increases we expect that coordination on 


\begin{tabular}{|c|c|l|l|c|}
\hline & & Percent & Percent & Percent \\
$\mathrm{S}$ & $\mathrm{T}$ & ST AG & HARE & OTHER \\
\hline- & 0 & 0.0 & 96.1 & 3.9 \\
\hline 2 & 1 & 0.0 & 87.7 & 12.3 \\
2 & 2 & 0.0 & 92.6 & 7.4 \\
2 & 4 & 0.0 & 96.1 & 3.9 \\
\hline 3 & 1 & 67.1 & 29.4 & 3.5 \\
3 & 2 & 86.5 & 11.7 & 1.8 \\
3 & 4 & 96.4 & 2.7 & 0.9 \\
\hline 4 & 1 & 90.4 & 7.9 & 1.7 \\
4 & 2 & 97.4 & 1.9 & 0.6 \\
4 & 4 & 99.4 & 0.4 & 0.3 \\
\hline
\end{tabular}

Table 2: Percent of total time spent in the various outcomes $(C=18.5)$.

(Stag,Stag) will become increasingly difficult.

To simplify the analysis we classify outcomes of the system into three mutually exclusive categories. We say that the system is in $S T A G$ if more than $60 \%$ of all games in a given generation end in (Stag,Stag). Similarly, we say that the system is in $H A R E$ if more than $60 \%$ of all games end in (Hare,Hare). If neither of the these conditions are met, we say that the system is in OTHER. Given these definitions, the more $S T A G$ observed over time, the better the agents are able to coordinate on the superior outcome.

Table 2 gives the percent of time the system spends in the three outcomes (when $C=18.5$ ). For each set of parameters the system was run for one million generations. ${ }^{4}$ In general the system tends to spend very little time in OTHER. With no communication, or when the automata have fewer than three states, the system spends the majority of its time in HARE. As the number of states increases beyond two, we see a sudden change in the behavior of the system and it begins to spend a significant amount of time in $S T A G$. Once agents possess three or more states, increasing tokens $(T)$ increases the percent of time the system spends in $S T A G$.

Thus, we see that communication promotes better outcomes, but only if agents are sufficiently sophisticated to use communication. Automata with less than three states are simply unable to employ any kind of sensible communication strategy, since there are not enough states available to the automaton to fully react to communication. Furthermore, having the ability to communicate and possessing less than three states appears to hinder coordination (even on (Hare,Hare)) relative to having no communication ability $(T=0)$ at all.

Figure 2 highlights the impact of the $C$ parameter on the model's behavior.

\footnotetext{
${ }^{4}$ We begin recording data after the first 100 generations to minimize the impact of any initial conditions. Moreover, for systems with greater than two states, we we find that given the dynamics the effects of any initial condition are quickly eroded - the data collected from one run of one million generations is not significantly different from that generated from ten runs, each of one hundred thousand generations.
} 


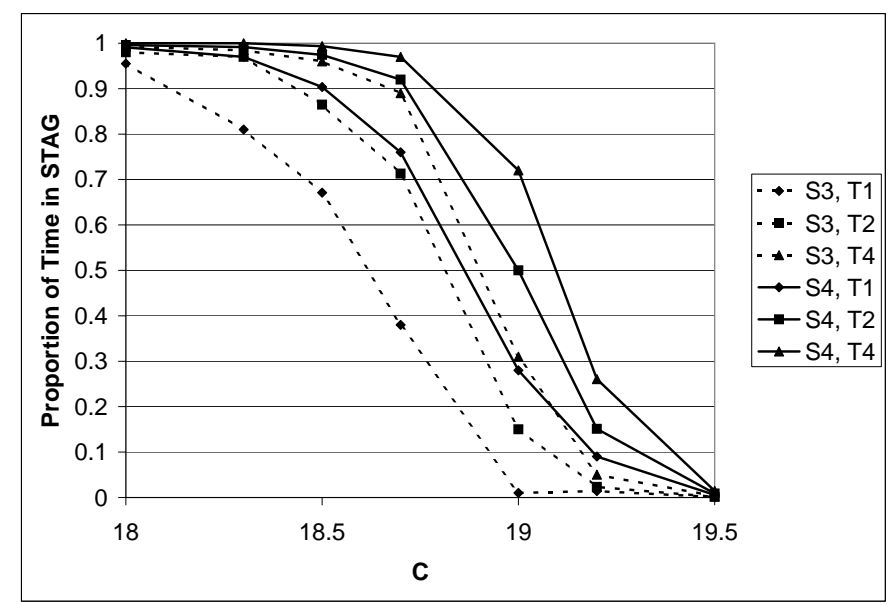

Figure 2: The impact of $C, S$, and $T$ on $S T A G$ coordination.

Recall that as $C$ increases, playing Hare versus Stag should become more attractive all else equal, which is indeed what we observe from the figure. Moreover, there appears to be a fairly systematic relationship between the effect of $S$ and $T$ on time spent in $S T A G$ across all values of $C$. In general, four-state agents (shown as solid lines in the figure) dominate (that is, result in more STAG) three-state agents (dashed lines), and more communication tokens dominate fewer tokens. As $C$ increases above 18 we first see that the variance of time in $S T A G$ increases across the various parameter settings (for example, when $C=19$ the amount of time spent in ST AG can range from around $0 \%$ to $70 \%$ depending on the parameters), and then, once $C$ goes much beyond 19, the system collapses to spending almost all of its time in $H A R E$ regardless of the other parameters.

\subsection{Dynamics}

The results of the previous section indicate that communication can promote better coordination among the agents in the system. In this section we investigate how such coordination comes about. To do so, we explore the dynamics of the system. In particular, we focus on how the system transitions from one coordination regime to another. Recall that the system tends to spend the majority of its time in either STAG or HARE (with occasional and limited forays into $O T H E R$ ). While the system concentrates on these two outcomes, it is not static, but rather it experiences frequent transitions between these outcomes. 


\begin{tabular}{|c|c|c|c|r|c|}
\hline & & TBT & TBT & \multicolumn{1}{c|}{ TIR } & TIR \\
$\mathrm{S}$ & $\mathrm{T}$ & STAG & HARE & STAG & HARE \\
\hline 2 & 1 &.-- & 10.2 &.-- &.-- \\
2 & 2 &.-- & 16.6 &.-- &.-- \\
2 & 4 &.-- & 29.8 &.-- &.-- \\
\hline 3 & 1 & 41.2 & 33.2 & 3289.6 & 1442.9 \\
3 & 2 & 76.7 & 70.4 & 5258.7 & 722.7 \\
3 & 4 & 146.4 & 89.0 & 15233.2 & 431.5 \\
\hline 4 & 1 & 86.2 & 75.3 & 6102.6 & 530.4 \\
4 & 2 & 223.7 & 115.6 & 16941.4 & 342.8 \\
4 & 4 & 497.5 & 92.4 & 33509.0 & 119.1 \\
\hline
\end{tabular}

Table 3: Mean consecutive time between transitions and in regime $(C=18.5)$.

To facilitate the analysis below we need to define a transition. Here we consider it a transition whenever the outcome (STAG, HARE, or OTHER) changes from one generation to the next. Thus, if the system is in, say, $S T A G$ at generation $t$ and goes to OTHER at $t+1$, we have observed a transition from $S T A G$ to $O T H E R$ (which we designate as a $S O$ transition to simplify notation). At times it is also useful to consider longer chains of transitions, such as when the systems moves from STAG to OTHER at time $t$, remains in $O T H E R$ for some time, and then transitions to, say, HARE at time $t+k$. In the analysis below, this would be considered a $S O H$ transition. Transitions that don't involve OTHER are extremely rare, and thus we ignore them in the discussion below.

Table 3 provides various measures of the average consecutive generations spent in $S T A G$ and HARE. The TBT (time between transitions) values provide the average number of consecutive generations the system spends in a given outcome between the transitions into and out of that outcome. The TIR (time in regime) values give the average consecutive generations spent in a given outcome ignoring any transitions that go to OTHER and return back to the same outcome. This latter measure smoothes out any transients that do not cause a major change in the coordination point. In general, $T B T$ increases with the number of tokens $(T)$ or states $(S)$, ceteris paribus. Thus, "better" communication appears to enhance the ability of the system to stabilize, at least in the short run, on a particular coordination point, regardless of which one. The TIR figures indicate that increases in states or tokens differentially favor the system locking into $S T A G$ rather than HARE regimes. By comparing TIR to TBT we can get a sense of the average number of transitions into OTHER that do not result in a coordination regime change. In general, $S T A G$ regimes sustain around 70 such transients before being broken, while $H A R E$ regimes tend to have fewer than 10 .

The transitions that have the largest impact on the behavior of the system are those that cause a shift in the coordination regime, that is, the HOS and 


\begin{tabular}{|l|l|c|l|l|l|l|}
\hline S & T & $\begin{array}{c}\text { Number } \\
\text { of } H O S \\
\text { Transitions }\end{array}$ & $\begin{array}{c}\text { Mean } S O S \\
\text { Transition } \\
\text { Time }\end{array}$ & $\begin{array}{c}\text { Mean } S O H \\
\text { Transition } \\
\text { Time }\end{array}$ & $\begin{array}{c}\text { Mean } H O S \\
\text { Transition } \\
\text { Time }\end{array}$ & $\begin{array}{c}\text { Mean } H O H \\
\text { Transition } \\
\text { Time }\end{array}$ \\
\hline 3 & 1 & 211 & 1.4 & 5.3 & 2.9 & 1.3 \\
3 & 2 & 166 & 1.3 & 5.5 & 3.1 & 1.3 \\
3 & 4 & 63 & 1.2 & 5.3 & 3.2 & 1.4 \\
\hline 4 & 1 & 150 & 1.4 & 5.5 & 2.5 & 1.3 \\
4 & 2 & 58 & 1.3 & 5.3 & 2.8 & 1.7 \\
4 & 4 & 29 & 1.3 & 6.1 & 2.6 & 2.3 \\
\hline
\end{tabular}

Table 4: Number of HOS transitions and mean transition time (in OTHER) among different types of transitions $(C=18.5)$.

$S O H$ transitions. The third column in Table 4 gives the observed number of $H O S$ transitions out of one million generations. In general, more states (and within a given number of states, more tokens) reduce the number of such transitions. The pattern for the number of $S O H$ transitions is identical, since for every HOS transition there must be an associated $S O H$ transition. ${ }^{5}$ Table 4 also gives the mean transition time between the key types of transitions. The mean time is measured as the time spent in OTHER between the two bounding outcomes. In general, $S O S$ and $H O H$ transition times are much shorter than $S O H$ and HOS times, often lasting only slightly more than one generation. Also note that $H O S$ transitions tend to take much less time than $\mathrm{SOH}$ ones (the former around two generations less). This difference in average transition time suggests that there may be different underlying mechanisms driving these transitions.

To improve our understanding of the various transitions, we examine the strategic dynamics. The number of unique strategies grows geometrically in $S$ and $T$, so to facilitate this investigation, we classify agents into six mutually exclusive categories. The first two categories capture agents that do not communicate at all and are unresponsive to the messages of others. NoCommStag and NoCommHare are used to designate agents of this sort that play Stag and Hare respectively. The second two categories capture agents that begin by communicating, but then, regardless of the observed messages, always play the same final move. Such strategies that always play Stag are classified as CommStag and those that always play Hare as CommHare. The fifth type of strategy, CommEither, begins by communicating and, depending on what is received, plays either Stag or Hare. Finally, Chatter strategies are those that communicate but have the potential of never reaching a final move.

Any strategy that arises in the system can be classified as one (and only one) of the above types. Note that this classification is very rough. For example, a

\footnotetext{
${ }^{5}$ To see this, note that to get two $H O S$ transitions you must have one $S O H$ transition that moves you back to $H A R E$ from $S T A G$. Thus, the number of $H O S$ and $S O H$ transitions can at most differ by one.
} 


\begin{tabular}{|c|c|ccc|c|}
\hline Agent Type & Base & $\begin{array}{c}\text { Pre- } \\
\text { Transition }\end{array}$ & $\begin{array}{c}\text { During- } \\
\text { Transition }\end{array}$ & $\begin{array}{c}\text { Post- } \\
\text { Transition }\end{array}$ & Base \\
\hline \hline & STAG & STAG & OTHER & STAG & STAG \\
\hline NoCommStag & 0.53 & 0.15 & 0.13 & 0.16 & 0.53 \\
CommStag & 0.34 & 0.52 & 0.44 & 0.50 & 0.34 \\
CommEither & 0.08 & 0.24 & 0.24 & 0.25 & 0.08 \\
CommHare & 0.02 & 0.04 & 0.09 & 0.04 & 0.02 \\
NoCommHare & 0.02 & 0.03 & 0.07 & 0.03 & 0.02 \\
\hline & STAG & STAG & OTHER & HARE & HARE \\
\hline NoCommStag & 0.53 & 0.21 & 0.12 & 0.03 & 0.02 \\
CommStag & 0.34 & 0.52 & 0.29 & 0.04 & 0.02 \\
CommEither & 0.08 & 0.19 & 0.18 & 0.08 & 0.04 \\
CommHare & 0.02 & 0.04 & 0.23 & 0.49 & 0.42 \\
NoCommHare & 0.02 & 0.03 & 0.16 & 0.36 & 0.48 \\
\hline \hline & $H A R E$ & $H A R E$ & OTHER & $H A R E$ & $H A R E$ \\
\hline NoCommStag & 0.02 & 0.03 & 0.06 & 0.03 & 0.02 \\
CommStag & 0.02 & 0.03 & 0.06 & 0.03 & 0.02 \\
CommEither & 0.05 & 0.08 & 0.10 & 0.08 & 0.04 \\
CommHare & 0.42 & 0.66 & 0.58 & 0.67 & 0.42 \\
NoCommHare & 0.47 & 0.18 & 0.13 & 0.17 & 0.48 \\
\hline & $H A R E$ & $H A R E$ & OTHER & STAG & STAG \\
\hline NoCommStag & 0.02 & 0.03 & 0.07 & 0.13 & 0.53 \\
CommStag & 0.02 & 0.03 & 0.10 & 0.17 & 0.34 \\
CommEither & 0.05 & 0.25 & 0.52 & 0.61 & 0.08 \\
CommHare & 0.42 & 0.17 & 0.13 & 0.07 & 0.02 \\
NoCommHare & 0.47 & 0.50 & 0.18 & 0.03 & 0.02 \\
\hline
\end{tabular}

Table 5: Mean distribution of population proportions of strategy types $(S=$ $3, T=2, C=18.5)$.

CommEither agent may end up in an endless cycle of communication with a particular opponent, since the classification is based only on the potential for the behavior, not its guaranteed realization. Nonetheless, however crude, the above categories are sufficient to capture some key behavior of the system.

Table 5 gives the average population distribution of the first five strategy types $^{6}$ at four critical periods. The "Base" period is anytime the system is either in STAG or HARE. The "During Transition" period is when the system is in OTHER during an actual transition . The "Pre-" and "Post-Transition" periods are the three periods just prior to entering and leaving, respectively, OTHER. ${ }^{7}$

The upper half of Table 5 gives data for $S O S$ and $S O H$ transitions. During

\footnotetext{
${ }^{6}$ The omitted category, Chatter, constitutes a very small part of the population.

${ }^{7}$ Occasionally, there are fewer than three such periods, in which case we use whatever periods are available.
} 
the Base and Pre-Transition periods, there are few differences in the distributions of agent types for either of these two types of transition. During the Base period, the most prominent strategy types are NoCommStag followed by CommStag. During the Pre-Transition period there is a growth of both CommStag and CommEither at the expense of the NoCommStag strategies. It is only at the During-Transition stage that differences between the two types of transitions become apparent. If the system returns to $S T A G$ (such $S O S$ transitions are shown in the upper half of the top panel), the population distribution changes little until it eventually moves back to the Base-period proportions. If, on the other hand, the system transitions into HARE (SOH transitions are shown in the lower half of the top panel), CommHare increases at the expense of CommStag, which eventually leads to the population in HARE. Thus, it appears that $S O H$ transitions are the result of relatively gradual shifts in population proportions.

The lower half of Table 5 shows the data for $\mathrm{HOH}$ and $H O S$ transitions. In the Base period, the population is dominated by both CommHare and NoCommHare types. In $\mathrm{HOH}$ transitions (upper half of the bottom panel), the major change observed is an increase in the CommHare types, mostly at the expense of NoCommHare, during the Pre-Transition period. These new proportions are maintained throughout the transition, but they are insufficient to move the system to a different coordination regime. The population dynamics of HOS transitions (lower half of the bottom panel) appear very different from the outset. In these types of transitions, the most notable change is a sharp rise in CommEither types. CommEither types constitute only $5 \%$ if the population in the Base period, rise to $25 \%$ during Pre-Transition, and eventually reach $61 \%$ of the population. Starting in the During-Transition period, CommStag types begin to increase in the population while the NoCommHare experience a rapid decline. As the system settles on the new coordination point, the proportion of CommEither falls from $61 \%$ to around $8 \%$ of the population.

The differences in population proportions across the various transitions hint at the mechanisms underling the dynamics of the system. First, note that HOS and $S O H$ transitions appear to be driven by very different mechanisms, as CommEither plays a key role in HOS transitions, but not in $\mathrm{SOH}$ transitions. Next we consider a highly stylized version of the model to develop an explanation for the above dynamics.

To understand the dynamics underlying the transitions it is helpful to consider some simple payoff relationships in a stylized version of the game. Suppose that there are only two types of strategies in the population: those that always play Stag and those that always play Hare. Let $\rho_{s}$ be the proportion of the population that plays Stag. Given the values in Table 1, the payoff to a Stag player, $\pi_{s}$, is equal to $20 \rho_{s}+10\left(1-\rho_{s}\right)$. Similarly, the payoff to a Hare player, $\pi_{h}$, is $C \rho_{s}+12\left(1-\rho_{s}\right)$. Therefore,

$$
\pi_{s}>=<\pi_{h} \Leftrightarrow \rho_{s}>=<\frac{2}{22-C} .
$$

Thus, the attractiveness of playing Stag (or Hare) is directly tied to a critical 
proportion, $\rho_{s}^{*}$, of players playing Stag. If this proportion is greater than $\frac{2}{22-C}$, then players should play Stag, otherwise they should play Hare. As $C$ increases $\rho_{s}^{*}$ increases, requiring a higher proportion of overall Stag play before it becomes the best action.

One plausible mechanism explaining transitions is random mutation. Even if all of the agents are playing Stag, if enough Hare players arise via mutation, the system can tip into playing Hare (and vice versa). For $20>C \geq 18$ we have $1.0>\rho_{s}^{*} \geq 0.5$ respectively, so mutation driven transitions will have an easier time moving the system from $S T A G$ to $H A R E$ than vice versa, and thus such a mechanism may underlie the $S O H$ transitions. This hypothesis is discussed more toward the end of this section.

Suppose that, in addition to pure Hare and Stag players, we add a strategy type that can discriminate in a limited way among its opponents. An obvious mechanism to implement such a strategy would be through communicationif my opponent communicates in the expected way then treat them as "self," otherwise treat them as "other." 8 Suppose that such a loquacious strategy arises in a world of all Hare and plays Stag with self and Hare otherwise. In this case, the strategy would do better than average as it either gets the (Hare,Hare) payoff when playing with others or the (Stag,Stag) payoff when playing with self, while its opponents only receive (Hare,Hare). Note that the loquacious strategy has an advantage only in the above scenario, as in all other possible configurations of the world (for example, a world of all Stag and a loquacious strategy that plays Stag with others and Hare with itself), it will never do better and typically it will do worse than its opponents.

The notion that the emergence of a loquacious strategy drives HOS transitions is consistent with our observations. As previously discussed, $H A R E$ regimes are dominated by NoCommHare and CommHare strategies. In such a world, these two types of strategies can coexist, as they both end up playing Hare against each other. Since they both receive the same payoff, the population proportions can easily "drift" between these two strategies. As noted above, HOS transitions are preceded by a sharp increase in CommEither agents (the proportion of such agents rises from $5 \%$ to $61 \%$ across the transition phases). We also observe a large number of NoCommHare agents in the Pre-Transition phase, so the conditions are not unlike the ones described in the stylized model above, in which the population is vulnerable to invasion by an "intelligent" strategy that can play Stag with itself and Hare otherwise. The low number of CommHare types in the Pre-Transition phase limits the scenario in which the CommEither agents get fooled by the conversation of the CommHare into playing Stag against Hare. Given the above conditions, the CommEither agents can take over the population quickly and cause a transition between coordination on Hare to coordination on Stag in only a few generations.

The importance of CommEither strategies to HOS transitions can be verified in a variety of ways. We find that large numbers of CommEither agents

\footnotetext{
${ }^{8}$ Note that the term self is meant to designate agents that behave in the same way, rather than the literal meaning of self.
} 
(here defined as over $35 \%$ of the population) are present in around $80-95 \%$ of the HOS transitions, with the likelihood of their presence increasing in both states and tokens. We suspect that the remaining transitions are driven by pure mutation events. An alternative test of the importance of CommEither agents to HOS transitions is to run an experiment in which we eliminated any CommEither agents that may arise in the population by replacing them with random, non-CommEither agents. We find that in such an experiment the number of HOS transitions drops to zero and the system locks into HARE.

Following a HOS transition, we observe the average proportion of CommEither agents falling to low levels. Thus, even though CommEither agents should not be payoff disadvantage in $S T A G$, their proportion in the population rapidly declines (for example, with $S=3, T=2, C=18.5$, this decline occurs in under eight generations). Since only selection and mutation act to alter the population from generation to generation, the cause of this decline can be tied to either one, or both, of these processes. A direct inspection of the average payoffs suggests that CommEither agents do not earn significantly less than other types of agents in the population following HOS transitions, so selection can not drive the decline. Thus, mutation appears to be the driving force.

The impact of mutation on the system is closely tied to the structure of the agents. The mutation operator is not "linear" in the sense that random changes in the structure of an automaton can have dramatically different consequences. For example, consider a NoCommHare strategy. Regardless of the size of the automaton or the various choices of actions and transitions, the key defining feature of such a strategy is that the action in the first state is to play Hare. Thus, unless mutation alters this particular action, it will not change the behavior of this strategy. Moreover, if mutation does modify that action to, instead, send a communication token, then a vast range of new strategic behaviors becomes possible depending on the configuration of the other states of the machine.

Knowing how vulnerable the various types of agents are to mutation will help us understand the observed decline of CommEither. Table 6 gives the estimated transitions when a randomly created three-state, two-token agent undergoes a single mutation. The values down the main diagonal of the matrix indicate that CommEither agents are more susceptible to transformation via mutation than the other agent types. In particular, around $36 \%$ of the time CommEither agents get transformed by mutation into some other agent type.

Thus, CommEither agents appear to play a key, but transient, role in getting the system to the better coordination point. In HOS transitions, CommEither agents are able to quickly invade the population and facilitate the system moving from $H A R E$ to $S T A G$ due to selective pressure. Notwithstanding this critical role, CommEither agents are so fragile that they cannot withstand the vagrancies of mutation, and their numbers rapidly dwindle in the post $H O S$ world.

We now turn our attention to why the system, once in $S T A G$, transitions back to HARE. Recall from Table 5 that CommEither agents do not appear to play a pivotal role in such transitions. This is not too surprising, since there are no payoff gains to such strategies in $S T A G$ and they are hard to maintain 


\begin{tabular}{|c|c|c|c|c|c|c|}
\hline & \multicolumn{7}{|c|}{ Post-Mutation Type } \\
\hline Pre-Mutation Type & & & & & & \\
& NoCommStag & CommStag & CommEither & CommHare & NoCommHare & Chatter \\
\hline NoCommStag & 0.88 & 0.02 & 0.01 & 0.02 & 0.04 & 0.03 \\
CommStag & 0.04 & 0.75 & 0.05 & 0.03 & 0.04 & 0.08 \\
CommEither & 0.04 & 0.14 & 0.64 & 0.14 & 0.04 & 0.00 \\
CommHare & 0.04 & 0.03 & 0.05 & 0.76 & 0.04 & 0.08 \\
NoCommHare & 0.04 & 0.02 & 0.01 & 0.02 & 0.88 & 0.03 \\
Chatter & 0.04 & 0.07 & 0.00 & 0.07 & 0.04 & 0.77 \\
\hline
\end{tabular}

Table 6: Estimated probabilities of strategic transformation for a randomly created strategy undergoing a single mutation $(S=3, T=2, n=1000$ per row).

in the absence of any selective pressure. Thus, there is an asymmetry in the potential for communication here, and some other mechanism must induce the transition.

Previously, we discussed how mutation has the potential to tip the system from one coordination point to another. If the proportion of Stag play drops to less than $2 /(22-C)$, then Hare becomes the best reply. Since $C \geq 18$, there is a bias in the system towards tipping from $S T A G$ to $H A R E$ all else equal. The ability of mutation to generate enough change in the strategies actually to tip the system is tied to the underlying population composition. As shown in Table 6, NoCommStag strategies are relatively invulnerable to mutation, so their presence will encumber tipping from $S T A G$ to $H A R E$. Indeed, Table 5 suggests that in the Pre-Transition period of $S O H$ transitions, there is a large shift in the population away from NoCommStag and into CommStag strategies. Since these two types of strategies receive the same payoff when playing one another, their proportions in the population can drift back and forth. When the proportion drifts toward CommStag, then the conditions are more favorable for mutation to generate a sufficient number of Hare players to tip the system into the inferior coordination point. Transitions that are driven purely by the initial accumulation and selection of unfavorable mutations should take longer than those (like $H O S$ ) driven by favorable selection, and this is consistent with the data (see Table 4).

The dynamic picture that emerges from the analysis above is illustrated in Figure 3. In this figure, each ball represents the current state of the population and mutation "bounces" the ball around the landscape. In general, there are two basins of attraction in this system, one in which the players coordinate on Hare and one in which they coordinate on Stag. The height of the wall between these two basins is shorter on the Stag side than on the Hare side, and thus a bouncing (mutating) ball will have an easier time jumping from Stag to Hare than vice versa. As $C$ increases, the Hare basin deepens while the Stag one becomes shallower, exacerbating the height difference between 


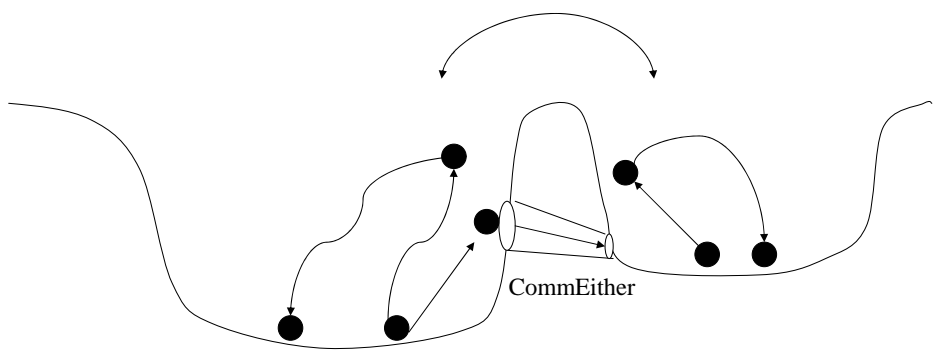

Hare Basin

Stag Basin

Figure 3: A simple physical analogy of the dynamics.

the two basins. Thus, under mutation pressure alone, the system will tend to the Hare equilibrium with occasional forays into Stag. However, the ability to communicate opens up a temporary "portal" that allows a ball in the Hare basin to tunnel under the separating wall and enter the Stag basin. This portal exists for only a moment in time when CommEither agents emerge in the population. Once the population enters the Stag basin, the portal - and even the need for communication - dissolves.

\section{Conclusion}

We find that communication can play a key role in the ability of agents to reach, and maintain, superior coordination. In the absence of communication, the system we analyzed tends to get trapped at the inferior coordination point. However, once agents reach a particular strategic threshold, communication allows the population to coordinate on the superior outcome. Note that agents were only allowed to send a priori meaningless messages and thus the meaning of the tokens had to arise endogenously via the decentralized interactions of the agents, shaped only by indirect, adaptive pressures taking place across a time scale of several generations. We also found that improving the potential for agents to implement more complicated strategies or increasing the number of possible communication tokens served to further enhanced the coordination.

While the system tends to spend large amounts of time at the various coordi- 
nation points, it is by no means static - periods of coordination are interrupted by rapid transitions in which the system switches to the alternative coordination point. We analyzed the various mechanisms that account for this dynamic behavior and found that there are a few critical pathways through which the system transitions from one coordination point to another. Occasionally, stochastic changes induced by the adaptive system will cause enough strategic disruption to tip coordination points. Such tips are more likely to be successful when the current population is composed of strategies that are more easily manipulated via mutation by the adaptive mechanism. Moreover, given our parameters, these types of transitions tend to favor movements from the superior to the inferior coordination point.

Communication plays a key role in an alternative pathway that drives the system from the inferior to the superior coordination point. Agents with the ability to communicate and differentiate signals sent by others serve as a catalyst that allows the system to rapidly transition out of the inferior coordination point. During these very short transition periods, these communicative agentsnormally present at very low background levels of $4-8 \%$ of the populationrapidly rise to over $50 \%$ of the population and then, as quickly, die off. Thus, while communication is vital to improving the outcome of the system, it is present for only a brief period of time.

The model used here (based on the work of Miller et al. [7]) provides a rather primitive setting in which to explore communication. Agents are quite limited in their ability to communicate with one another and process what is said. Moreover, strategies are only modified by very simple adaptive mechanisms. Thus, the model serves as a nice lower-bound for understanding the potential of communication to emerge and alter social systems.

As shown above, even primitive forms of communication have the ability to expand the behavioral repertoire of agents in productive ways. In both the above work on coordination and previous work on cooperation, communication allowed the emergence of agents that could begin to differentiate interactions with other agents in the system. Such differentiation allowed such agents to exploit the potential of the better outcome (once partners were suitably identified) while also maintaining the ability to effectively compete with agents trapped in the inferior outcome. Notwithstanding standard theoretical arguments about cheap talk to the contrary, in simple adaptive systems - where agents do not instantly adjust to the long-term implications of deductive reasoning - dynamic cascades of agent behavior have the potential to lead the system into previously unattainable goals. Once these goals are achieved, the value of communication may diminish. In the case of cooperation, mimics arose and destroyed the benefit of communication; in the case of coordination, once the new outcome was achieved communication was no longer necessary and evolutionary forces (mutation) caused communication to atrophy.

We also found that improving the potential for communication improves the performance of the agents. Under conditions favorable to both coordination and cooperation, increasing the potential of agents to implement more complicated strategies (via automaton states) or the potential of the underlying 
communicative system (via available communication tokens) promotes better outcomes. While there is still work to be done on elucidating the actual mechanisms underlying these results, we suspect that increases in the potential for communication allow rich and robust "ecologies" of strategies to emerge in the model. We hypothesize that in such ecologies productive communicative pathways are much more likely to arise (important to coordination) and be sustained (important to cooperation).

The emergence of communication in the above model was predicated on agents having the potential, however primitive, to send, receive, and act on communication. This obviously leaves open the question of how such a potential can arise. In our model, sending a token or deciding on a final move in the game are both possible actions for an agent, with the only real difference between the two being that tokens do not directly alter the world and provide payoff. As such, it is not hard to imagine a continuum of action types between "tokens" and "final moves," in which communication emerges as "final move" actions become "tokenized" over time. For example, a gesture like raising up one's hands could be a final move that shows you have no weapons or, in a tokenized form, be used to indicate that you give up in frustration.

The ability of agents to communicate is likely to be a key mechanism by which agents, whether they be molecules, organisms, or economic entities, can improve their performance in interactive worlds. The model developed here suggests that even with simple adaptive mechanisms, endogenous systems of communication can easily arise and facilitate such activity. 


\section{References}

[1] Robert J. Aumann. Economic Decision Making: Games, Econometrics and Optimisation, chapter Nash-Equilibria are not Self-Enforcing. Elsevier, 1990.

[2] Gary Charness. Pre-play communication and credibility: A test of Aumann's conjecture. Universitat Pompeu Business, Economics Working Papers(293), 1998.

[3] Kenneth Clark, Stephen Kay, and Martin Sefton. When are nash equlibria self-enforcing? an experimental analysis. International Journal of Game Theory, 29(4):495-515, 2001.

[4] Russell Cooper, Douglas. V. DeJong, Robert Forsythe, and Thomas W. Ross. Communication in coordination games. Quarterly Journal of Economics, 107(53):739-771, 1992.

[5] Joseph Farell and Mathew Rabin. Cheap talk. Journal of Economic Perspectives, 10:103-118, 1996.

[6] John C. Harsanyi and Reinhard Selten. A General Theory of Equilibrium Selection in Games. MIT Press, 1988.

[7] John H. Miller, Carter T. Butts, and David Rode. Communication and cooperation. Jounal of Economic Behavior and Organization, 47:179-195, 2002. 